\title{
NGC 2477: Photometry and luminosity function ${ }^{\star, \star \star}$
}

\author{
G. Galaz ${ }^{1,4}$, M.T. Ruíz ${ }^{2,4}$, I. Thompson ${ }^{3}$ and M. Roth ${ }^{3}$ \\ 1 CNRS, Institut d'Astrophysique de Paris, 98 bis, Boulevard Arago, 75014 Paris, France \\ 2 Departamento de Astronomía, Universidad de Chile, Casilla 36-D, Santiago, Chile \\ 3 The Observatories of the Carnegie Institution of Washington, 813 Santa Barbara street, Pasadena, CA 91101-1292, \\ U.S.A. \\ 4 Visiting Astronomer, Las Campanas Observatory
}

Received January 30; accepted March 12, 1996

\begin{abstract}
Trough photometric methods we have constructed the luminosity function for the southern open cluster NGC 2477 down to $M_{v} \approx 12$. We present $R-I$ color-magnitude diagrams and near-infrared color-color diagrams, which allow us to separate statistically the cluster stars from the field ones, numerous because of the galactic latitude of the cluster. Our luminosity function (LF) reproduces some features obtained by other authors, namely, a dip at $M_{v} \approx 7$, and a probable maximum at $M_{v} \approx 12$. The method developed to separate cluster stars from field stars could be of interest to other astronomical problems.
\end{abstract}

Key words: open clusters: NGC 2477 — stars: luminosity function, mass function

\section{Introduction}

Among all the issues about Galactic structure and star count analysis we can find in the literature, one of the most recurrent is whether the luminosity function (LF) estimated from the stellar sample in the immediate neighborhood of the Sun (i.e. up to $50 \mathrm{pc}$ ), is the same as the one obtained for galactic clusters. A positive answer, implies that the fragmentation in the first stages of the stellar formation follows the same pattern, independently of the environment in which the stars had formed. Some authors have pointed out that the main sequence LF in some open clusters is consistent with a Salpeter (spectral index $=1.55$ ) initial mass fuction (IMF), (Carraro \& Ortolani 1994; Will et al. 1995) that is to say, the stellar population is dominated by relatively low-mass stars, i.e. $\leq 0.3 M_{\odot} \sim$ $55 \%$ (Aspin et al. 1994). On the other hand, other authors (Leggett et al. 1994) present results that, compared with theoretical models, indicate that the present cluster mass function is flat for masses less than $0.3 M_{\odot}$, implying that substellar objects make up no more than approximately $10 \%$ of the total mass of the cluster. At the same time, some models of stellar formation predicts that the num-

Send offprint requests to: G. Galaz (galaz@iap.fr)

${ }^{\star}$ Based on observations collected at Las Campanas Observatory in Chile, which is operated by The Carnegie Institution of Washington

${ }^{\star \star}$ Table 6 only available in electronic form at CDS via anonymous ftp 130.79.128.5 ber of substellar objects formed rises quickly at first, but then levels off to a nearly constant value, which it maintains until the dispersal of the cloud complex. Therefore, the number fraction of substellar objects always decreases with time (Fletcher \& Stahler 1994).

Here we present a photometric study of the open cluster NGC 2477 visible in the southern hemisphere. The cluster is located in the constellation Puppis $(\alpha$ (1950.0) $=7^{\mathrm{h}} 48^{\mathrm{m}}, \delta(1950.0)=-38^{\circ} 17^{\prime}, l=253.58^{\circ}$ and $b=$ $\left.-5.83^{\circ}\right)$ and is one of the richest cluster that can be found in the southern sky. Its age $(\sim 1.3 \mathrm{Gyr})$ and its richness are two features that make this cluster one of the most interesting to study among the old population Galactic clusters (Janes \& Phelps 1994). Recently, von Hippel et al. (von Hippel et al. 1995) have obtained deep HST images for the cluster, reaching $V \approx 26$, which allowed them to calculate the age of the cluster using white dwarfs' cooling models. The age which they found is consistent with the one used in this work. The population of old, "thin disk" clusters represents a unique sample of "test particles" for probing the galactic age-metallicity relation, the metallicity gradient, and the earliest stages of the formation of the galactic disk. The LF of NGC 2477 is poorly known and almost all the work has been done more than 20 years ago and therefore the photometry, although of good quality, is not very deep. The work of Hartwick et al. (1972), is among the most complete ones on NGC 2477. The distance, color excess and age used in this publication were taken from that work. We did photometry of NGC 2477 down to $R$ 
$\approx 23$ as well as in the near-infrared bands. Our principal aim is to achieve the faintest possible limiting magnitude $(I \approx 23)$, in order to reach the maximum in the LF, which have been observed by other authors to be near $M_{v}=12$ (Kroupa et al. 1992). One of the questions still not settled, is whether the LF is universal or dependent on the environment (the metallicity of the molecular cloud which formed the cluster, magnetic fields, etc). It seems that some features of the LF, recursively reported by different authors, are universal, namely, the gap observed near $M_{v}$ $=7$ (know as Wielen's dip; Wielen 1971) and the wellknown maximum near $M_{v}=12$. Kroupa et al. (1992), have interpreted those characteristics as a consequence of the mass-luminosity relation, and more precisely, the inflexion points of this relation near those magnitudes. Nevertheless, the discussion is still open and too many issues are not yet clear. Here, we explore the NGC 2477's LF confirming both the gap at $M_{v} \approx 7$ and the maximum at $M_{v} \approx 12$, even though we have observed only an area of $5 \operatorname{arcmin}^{2}$ of the whole $\sim 20 \operatorname{arcmin}^{2}$ of NGC 2477.

The paper is organized as follows: In Sect. 2 we explain the observations and reductions, in Sect. 3 we show the results of the photometry, previous step for the construction of the LF, which is explained in Sect. 4. In Sects. 5 and 6 we explore the presence of white dwarfs and low-mass stars, respectively, which could be present in our data. In Sect. 7 we summarize our main goals and discuss some specific points.

\section{Observations and reductions}

The observations were obtained during two runs at Las Campanas Observatory (LCO), with the $2.5 \mathrm{~m}$ DuPont telescope. In January 1992 the optical observations were carried out and in January 1993 the near-infared ones. In the optical run, a two-binned $2048^{2}$ Tektronix 2 CCD, with $R$ and $I$ Gunn filters were used (Thuan \& Gunn 1976), with a scale of $0.23^{\prime \prime} /$ pix, covering an area of 5 $\operatorname{arcmin}^{2}$. The region chosen for the observations was near the periphery of the cluster, centered at equatorial coordinates $(2000.0) \alpha=07^{\mathrm{h}} 52^{\mathrm{m}} 15^{\mathrm{s}}$ and $\delta=-38^{\circ} 32^{\prime} 39^{\prime \prime}$.

Images of five minutes exposure time in $R$ and $I$ were taken, as well as short exposure time images $(\approx 30 \mathrm{~s})$ of the same region of the cluster in order to include the brightest main sequence stars and giants. Also, exposures of a control field region close to the cluster $(\alpha(2000.0)=$ $\left.07^{\mathrm{h}} 52^{\mathrm{m}} 07^{\mathrm{s}}, \delta(2000.0)=-38^{\circ} 02^{\prime} 50^{\prime \prime}\right)$ were obtained. Some images were rejected because of either guiding problems or bleeding features. In total, nearly $90 \%$ of the total number of images taken were considered in the analysis (23 in $R$, 18 in $I$ ).

For the $R$ and $I$ photometric calibrations a series of 15 Landolt standards were observed at the beginning and at the end of each night (Landolt 1992). Care was taken to select the standard stars in order to include a wide range of colors $(-0.1 \leq R-I \leq 1.3)$ and magnitudes (12.0 $\leq R \leq 17)$. Image reductions for the calibrations were performed using IRAF ${ }^{1}$ packages CCDPROC, PHOT and PHOTCAL.

Near-Infrared $(J, H$ and $K)$ observations of the same region of the cluster were also made with a NICMOS3 (Rockwell) $256^{2} \mathrm{HgCdTe}$ photodiode array, with a scale of $0.33^{\prime \prime} /$ pix, each image covering an area of $1.44 \operatorname{arcmin}^{2}$. Because of the different coverages of the NICMOS3 detector with respect to the Tek2 CCD, the corresponding area covered by the optical data ( $R$ and $I$ filters), was divided into 9 subzones, each of them are covered by the NICMOS3 detector.

In order to calibrate the near-infrared magnitudes and colors of the program stars, a set of nine stars from the Infrared Faint Standard System (CalTech), were observed. The same stars were exposed at the beginning and at the end each night.

\section{Photometry}

Once the transformation to the standard system in each filter is done, we found the standard magnitudes for all stellar objects in the frame using as secondary standards the giants stars catalogued by Hartwick et al. 1972, included in the frames. In order to detect very faint stars, 23 frames in the $R$ band and 18 frames in the $I$ band (five minutes exposure time each) were aligned and averaged with a $3 \sigma$ rejection criteria, totaling $6900 \mathrm{~s}$ of exposure time in $R$ and $5400 \mathrm{~s}$ in $I$. This ensures that the frames are free of cosmic events. The same procedure was done for the $R$ and $I$ frames of the control field. In this way, each final frame for the cluster and the control field area $R$ and $I$ has a standard deviation for the sky $\sigma \approx 0.07$. The final image in I can be seen in Plate 1. Photometric reductions were carried out using DAOPHOT (Stetson 1987). Figure 1a show the color-magnitude diagram for the final list of stars with photometric errors less than 0.1. Figure $1 \mathrm{~b}$ shows the same for the control field region. In figure $1 \mathrm{a}$ the position of the seven cluster giant stars stand out in the frame (see Plate 2). Table $1^{2}$ shows some information about these giant stars and their name from the work of Hartwick et al. (1972). Five of the seven giant stars that appear in the diagram (region I), had been catalogued by Hartwick et al. (1972), the other two G1 and G2 were not because they are outside the cluster zone studied by those authors. These two additional stars G1 and G2 are at the same locus as the other five making them very likely cluster's giants.

Another feature seen in Fig. 1a, is the group of stars in the interval from $R \approx 12(I \approx 11)$ up to $R \approx 19(I \approx 18)$,

\footnotetext{
${ }^{1}$ Image Reduction and Analysis Facility, which is distributed by National Optical Astronomy Observatories (NOAO).

${ }^{2}$ The first $I D$ number is from this work and the $I D(\mathrm{H}-\mathrm{H})$ is referred to the work of Hartwick et al. (1972). G1 and G2, this work. The giants can be seen in Plate 2 .
} 


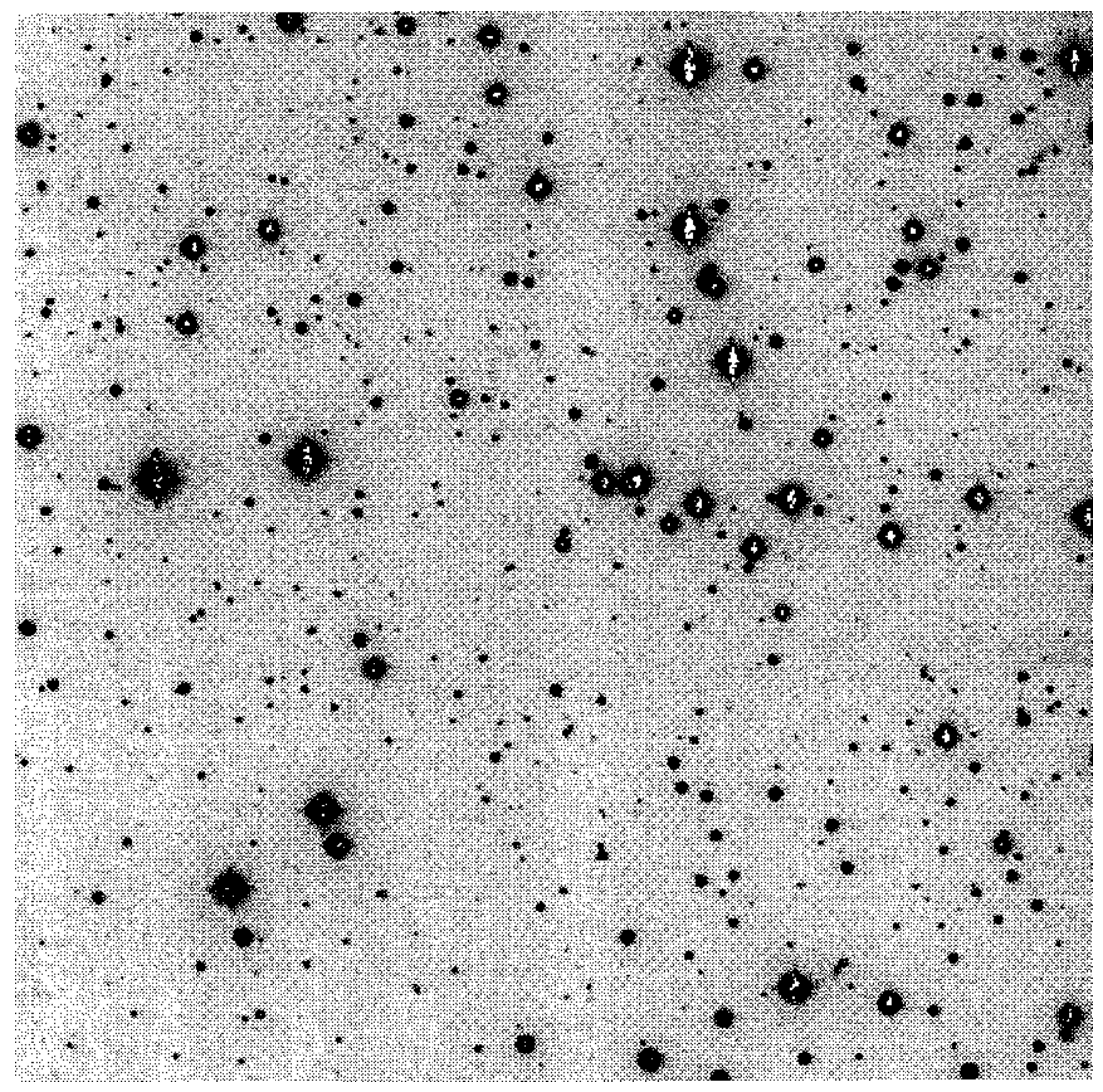

Plate 1.

Fig. 1. a) Color-magnitude diagram obtained in this work. Filters are the Thuan-Gunn $R$ and $I$. Here only the stars with errors in the color $R-I$ less than 0.1 magnitude have been plotted and taken into account

which are upper main sequence stars. From magnitude $R \approx 19(I \approx 18)$ this group begins to separate into two branches: one falls almost vertically down to magnitude $R \approx 24.5(I \approx 23)$, and the other falls diagonally to the right to magnitude $R \approx 25(I \approx 24)$. Between magnitudes $R \approx 22(I \approx 21)$ and the faintest magnitudes limit, the
Fig. 1. b) Color-magnitude diagram for the region used as control field. Also here, as in Fig. 1b, only stars with color errors smaller than 0.1 magnitude are shown

spread is large and the separation between both branches is less evident.

Once the positions of the stars are improved with ALLSTAR (DAOPHOT task), they are transformed from the CCD coordinates to the coordinates of the NICMOS3 detector in order to proceed to do the photometry in the $J, H$ and $K$ bands. In general, coordinate transformations 


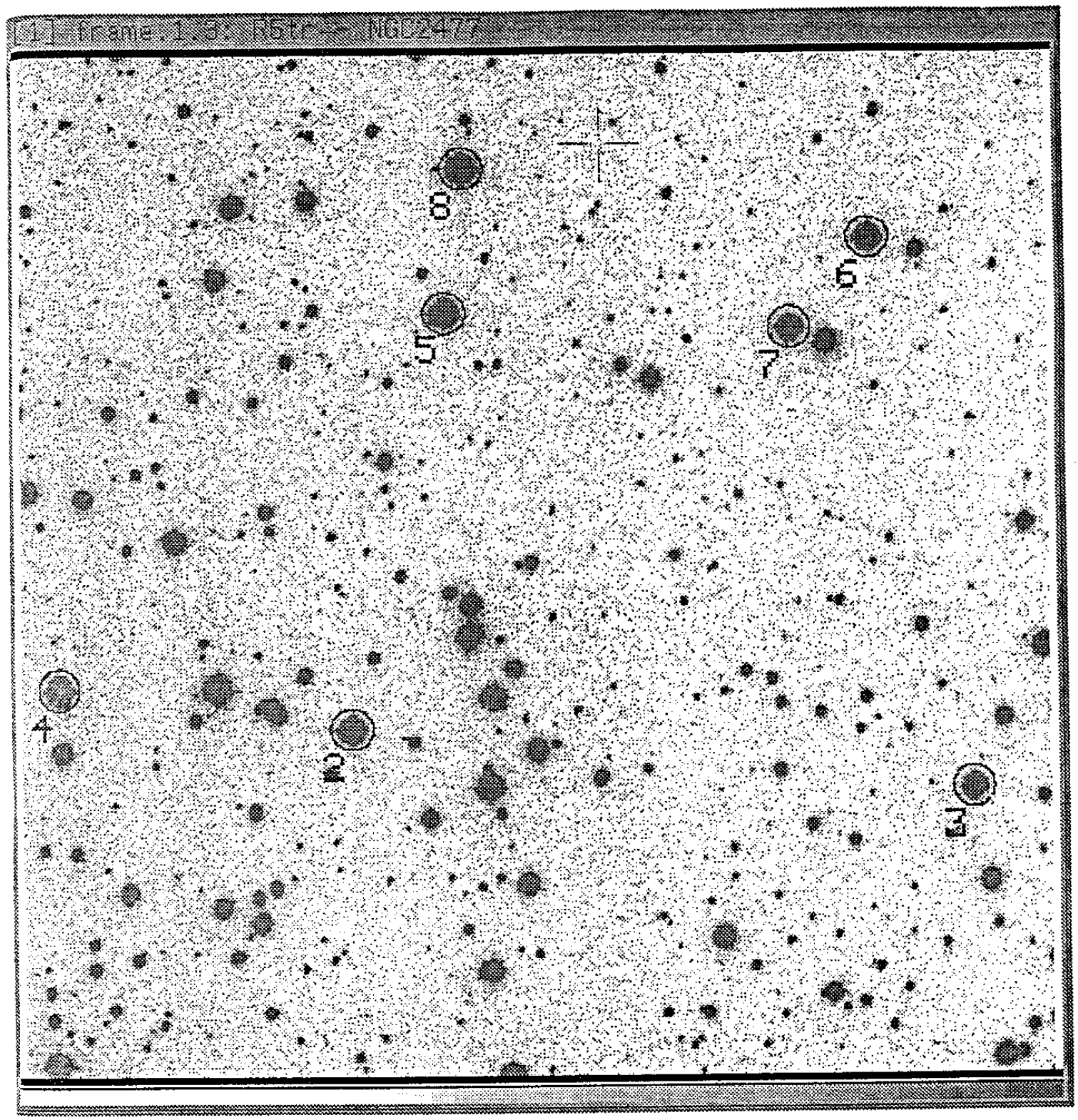

Plate 2.

Table 1. Magnitude and colors for cluster's giants

\begin{tabular}{ccccccc}
\hline$I D$ & $I D(\mathrm{H}-\mathrm{H})$ & $R$ & $I$ & $R-I$ & $V$ & $B-V$ \\
\hline 3 & $\mathrm{e}$ & 12.143 & 11.537 & 0.526 & 12.76 & 1.22 \\
2 & 7064 & 11.959 & 11.404 & 0.475 & 12.51 & 1.19 \\
4 & 8033 & 11.802 & 11.239 & 0.483 & 12.32 & 1.29 \\
5 & 8039 & 11.673 & 11.098 & 0.495 & 12.17 & 1.18 \\
8 & $\mathrm{I}$ & 11.393 & 10.801 & 0.512 & 12.05 & 1.22 \\
7 & $\mathrm{G} 1$ & 12.160 & 11.547 & 0.533 & & \\
6 & $\mathrm{G} 2$ & 11.956 & 11.331 & 0.545 & & \\
\hline
\end{tabular}

are well correlated, in both detectors, in a $97 \%$ confidence level. However, the fact that the limiting magnitude is no longer the same in all filters, produces that some stars do not appear in all bands. The band in which the faintest visual magnitude is reached is the $I$ band. For that reason, the initial positions for all the stars came from the $I$ filter. IR color-color and color-magnitude diagrams showed a very large scatter that prevented its use as a tool to separate cluster members from field stars. However, the combination of IR magnitudes with optical ones provided a very powerful way to separate these two different populations. Figures $2 \mathrm{a}$ and $2 \mathrm{~b}$ show color-color diagrams for the cluster. There, three different groups of stars are readily apparent, in particular for the $I-J / R-I$ diagram, which correspond to different regions of the HR diagram. Region I represents the giants. Regions II, III and IV (shown in the color-color diagrams), are the luminous stars of the upper main sequence of the cluster, the field stars in the line 


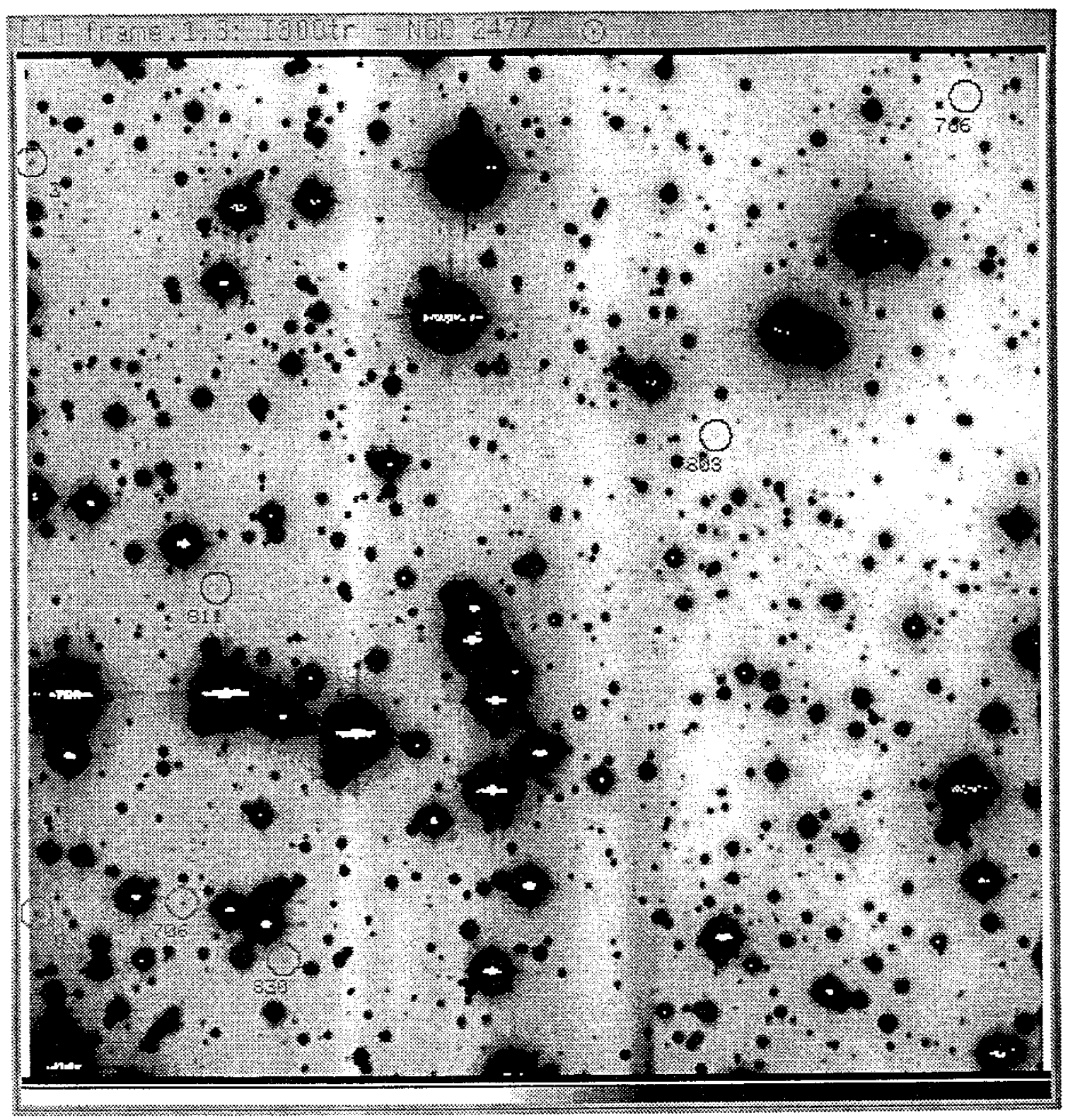

Plate 3.

of sight, and the rest of the main sequence stars, respectively. The last region contains the faintest and reddest stars of the cluster. In the next sections we will show that these different groups are not coeval, which will help us to extract the most probable cluster members to construct the cluster's LF.

\section{The luminosity function}

The main problem found in constructing the LF for the cluster was to distinguish among cluster and field stars. Once this task has been done with confidence, the construction of the LF is direct. The traditional way to perform this is to use kinematic data (i.e. proper motions and radial velocities), to separate the cluster members from the field stars. This can be done for clusters up to a few hundred parsecs, but further away this method fails. Here we use IR and optical photometry combined in colors to discriminate between cluster stars and field stars. From the $R$ vs. $R-I$ diagrams of Figs. $1 \mathrm{a}$ and $1 \mathrm{~b}$, in general one can observe a very different distribution of stars for the field frames (Fig. 1b) and the cluster + field frames (Fig. 1a), however it is hard to clearly separate them. This can be achieved using the color-color diagram $(I-J)$ vs. $(R-I)$. In Figs. $2 \mathrm{a}$ and $2 \mathrm{~b}$ we present the colors for the cluster + field frames. Three different groups can be distinguished, that have been marked II, III and IV in Fig. 2a, where they are clearer than in Fig. 2b. In order to identify these groups, with known populations in the more familiar $R$ vs. $(R-I)$ diagram, we identify the different stars 
Fig. 2. a) Color-color diagram for the cluster region. Region II shows the loci of stars brighter than $R \approx 17$. Region III shows the stars for which we make the hypothesis that they are field stars, and region IV shows the region of the CMD fainter than $R \approx 17$, with a higher probability to belong to the cluster. See also Figs. $3 \mathrm{a}$ and $3 \mathrm{~b}$, to see a precise correlation with the CMD

Fig. 2. b) Colors $R-I$ vs. $J-H$. Here, the scatter is not only large, but also the brightest stars of the cluster do not lie in a different loci

selected from the $(I-J)$ vs. $(R-I)$ diagram in the $R$ vs. $(R-I)$ one. The results are shown in Fig. 3a from where we can say that group II are upper MS cluster's stars. Figure $3 \mathrm{~b}$ shows the stars in group III of Fig. 2a, which indicates that group III are field stars. Group IV are then the lower main sequence cluster members. In order to separate statistically the cluster stars from the field ones, we have divided the $R$ vs. $(R-I)$ color-magnitude diagram in bins of 1.0 in magnitude and 0.1 in color, both for the cluster and the field stars region. Then, if we assume that the field stars, in the direction of the cluster, fill the same region in the color-magnitude diagram as the field stars in the field regions, but with different density, then the number of probable cluster stars for every magnitude bin

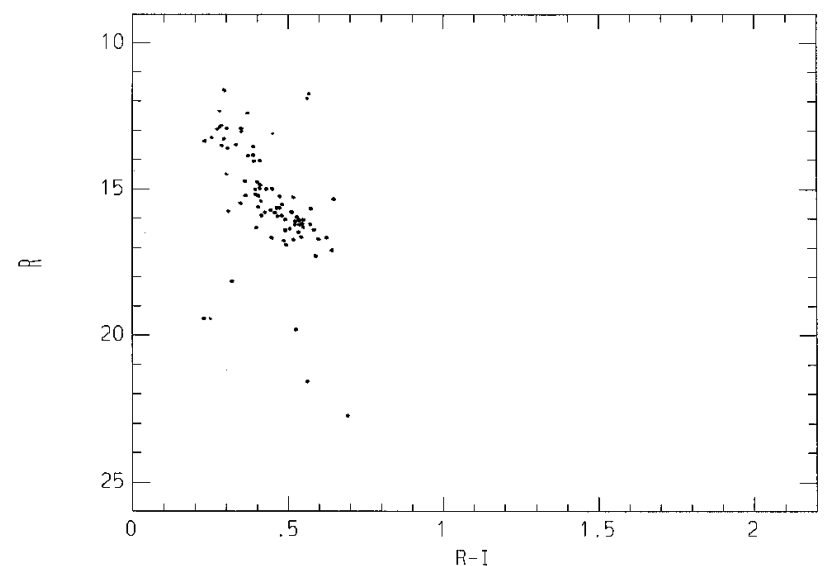

Fig. 3. a) CMD for the stars which correlate with the stars selected from the region II in Fig. 2a. These stars correspond mainly to the brightest stars of the cluster main sequence. Although stars of the region III have the same color $R-I$ (see Fig. 2a), they do not appear in this diagram, as we can see in Fig. 3b

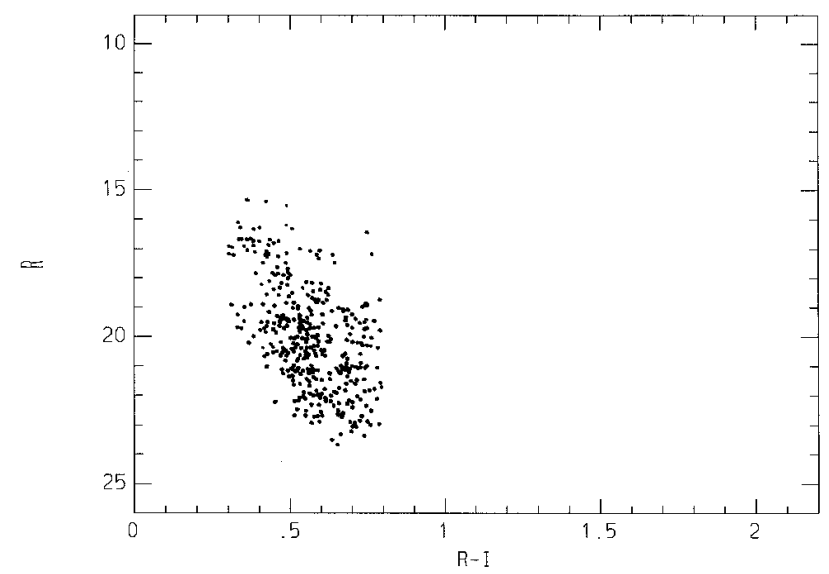

Fig. 3. b) CMD for the stars which correlate with the stars selected from the region III of the $R-I / I-J$ diagram. As we can see, these stars correspond mainly to field stars, following arguments explained in the text

$j$, is:

$$
\Psi_{j}=\sum_{i} \psi_{i j}
$$

where,

$$
\psi_{i j}=N_{i j}^{\mathrm{c}}-\xi N_{i j}^{\mathrm{f}} .
$$

In the last equations, $N_{i j}^{\mathrm{c}}$ is the number of stars in the color-magnitude cell $(i, j)$ in the cluster color-magnitude diagram and $N_{i j}^{\mathrm{f}}$ is the same but for the color-magnitude diagram of the control field region. The weighting parameter $\xi$ has to be used in order to scale the field stars density ${ }^{3}$

\footnotetext{
${ }^{3}$ Density in the color-magnitude plane.
} 
from the control field region, to the cluster region, i.e.,

$$
\xi=\frac{N^{\mathrm{f}}(\text { cluster region })}{N^{\mathrm{f}}(\text { field region })} \text {. }
$$

In Eq. (3), $N^{\mathrm{f}}$ (cluster region) is the number of stars that appear in the most probable loci of field stars, between the colors $R-I=0.3-0.6$ and magnitudes $R \approx$ $19-23$. In the same way, $N^{\mathrm{f}}$ (field region), is the number of stars in the color-magnitude diagram for the control field region, in the same color-magnitude loci. Note that the difference in Eq. (1) is performed in each cell $(i, j)$.

Other authors (see for example Fahlman et al. 1989), have performed the difference only for each magnitude bin. This is good enough to calculate the LF inside globular clusters, where the contamination by field stars, in the line of sight, is smaller than in open clusters, located near the galactic plane.
Fig. 5a for a 1.0 magnitude bin and in Fig. 5b for a 0.5 magnitude bin.

In order to produce a LF that could be compared to other works, it is necessary to have some information to link our data to the same magnitudes used by other authors. In this case we must transform our $R$ or $I$ magnitudes to $V$ magnitudes, which is the classical band used to construct the LF. We have identified some of the stars measured by Hartwick et al. (1972) and have obtained the magnitudes for those stars, which appear in our data. Our $R$ magnitude and $R-I$ color for such stars are shown in Fig. 6a. The color-color diagram $(B-V)$ vs. $(R-I)$ is shown in Fig. 6b. With these stars we correlate our $R$ and $I$ magnitudes with the $V$ magnitudes that appear in the work of Hartwick et al. (1972). The relationships are

$$
\begin{aligned}
& R=0.73+0.92 \mathrm{~V} \quad \sigma=0.07 \\
& V=-0.57+1.07 R \quad \sigma=0.09 \\
& I=1.23+0.87 \mathrm{~V} \quad \sigma=0.10 \\
& V=-0.92+1.12 I \quad \sigma=0.15 \text {. }
\end{aligned}
$$

Finally, to transform visual apparent magnitudes from the last equations, to absolute magnitudes, we use a distance modulus to NGC $2477(m-M)_{0}=10.61 \pm 0.21$ $(1331 \pm 128 \mathrm{pc})$, and a color excess $E_{B-V}=0.3$. With the aid of the work of Cohen et al. (1981) we calculate the extinction coefficients $A_{R}, A_{I}, A_{J}$ and $A_{K}$ (see Tables 2 and 3$)$.

Table 2. Extinction factors used in this work for the open cluster NGC 2477

\begin{tabular}{cc}
\hline Factor & value \\
\hline$A_{V}$ & 0.92 \\
$A_{R}$ & 0.70 \\
$A_{I}$ & 0.55 \\
$A_{J}$ & 0.24 \\
$A_{H}$ & 0.14 \\
$A_{K}$ & 0.08 \\
\hline
\end{tabular}

Fig. 4. Synthetic CMD contructed following Eq. (2). The number of stars which remains after the subtraction involved in Eq. (2) (and performed for each color-magnitude cell) is represented by the corresponding dots, distributed at random, for each cell. Here the weighting parameter $\xi$ (see Eq. (3)) is 0.91 . The bulk of points which remain at the left, for magnitudes fainter than 20, does not change the profile of our LF

The weighting factor $\xi$ is obtained empirically using Eq. (3) for the representative region of the field stars, which is defined as the region between $R-I \approx 0.3-0.6$ and $R \approx 19-23$. In principle, different $\xi$ can be calculated, but the differences among them are not large $(\approx 5-10 \%)$, preventing contrasting results in the star count analysis.

With this technique, it is possible to construct different synthetic color-magnitude diagrams which represent the subtraction involved in the Eq. (2). Figure 4 shows one of these diagrams for one value of $\xi$. The number of points in each color-magnitude cell is the number of stars that remain after the subtraction cluster-field (Eq. (2)) in the respective cell, the final number is distributed at random in each cell. The resulting number of stars for each magnitude bin is given by Eq. (1) and it is shown in
Table 3. Color excesses used in this work for the open cluster NGC 2477

\begin{tabular}{cc}
\hline Color Excess & value \\
\hline$E_{B-V}$ & 0.30 \\
$E_{R-I}$ & 0.15 \\
$E_{J-H}$ & 0.10 \\
$E_{H-K}$ & 0.06 \\
$E_{J-K}$ & 0.16 \\
\hline
\end{tabular}

The LF in visual magnitude constructed for the cluster from the synthetic color-magnitude diagram is shown in 
Fig. 7. A weighting factor $\xi=0.78$ was used. We can see a dip at $M_{v} \approx 7$ and a maximum at $M_{v} \approx 12$. We will show that completeness correction does not modify this result. The derived LF does not show a marked increase in the number of low luminosity stars in accordance with results from the star count works by Bahcall et al. (1994) and Paresce et al. (1995) work on the globular cluster NGC 6397.

Among the most important corrections to be considered in the observational star count analysis, are the completeness corrections, that have to be applied in order to perform meaningful comparisons between the observed and the theoretical LF. In the above section, the introduction of the weighting factor $\xi$ allows us to correct the incompleteness arising from the contamination of field stars. A second but also important correction can be performed by knowing the non-detectable stars' fraction. This is done by testing the degree of completeness of our data by means of simulations with artificial stars. For this task, we have used ADDSTAR routine inside DAOPHOT package (Stetson 1987), which introduces a suitable number of artificial stars $(\sim 10-15 \%)$ per magnitude bin in each original frame. Then, we reduce those frames by the same procedure described in the last sections. The ratio between the number of inserted and recovered stars gives an estimate of the incompleteness factor $f_{i}$, which is different for the cluster and for the field region. Table 4 shows the factor $f_{\mathrm{i}}$ for the final $R$ image, for the cluster and for the control field region. Up to apparent magnitude $R \approx 16$, all the inserted stars are recovered. For magnitudes fainter than 22.5, more than $50 \%$ are not detected.

Table 4. Incompleteness factors $f_{\mathrm{i}}$ relative to the frame $R$

\begin{tabular}{ccc}
\hline$R$ & $f_{\mathrm{i}}$ (cluster) & $f_{\mathrm{i}}$ (field) \\
\hline 10.50 & 1.00 & 1.00 \\
11.50 & 1.00 & 1.00 \\
12.50 & 1.00 & 1.00 \\
13.50 & 1.00 & 1.00 \\
14.50 & 1.00 & 1.00 \\
15.50 & 1.00 & 1.00 \\
16.50 & 1.00 & 1.00 \\
17.50 & 1.00 & 1.09 \\
18.50 & 1.18 & 1.12 \\
19.50 & 1.24 & 1.15 \\
20.50 & 1.27 & 1.17 \\
21.50 & 1.33 & 1.22 \\
22.50 & 2.13 & 1.70 \\
23.50 & 2.00 & 1.41 \\
24.50 & 3.10 & 1.55 \\
25.50 & 3.57 & 2.82 \\
\hline
\end{tabular}

These results have a direct impact in the LF of Fig. 7. To construct the corrected one, we multiply each output of Eq. (1) by the interpolated incompleteness factor $f_{\mathrm{i}}$. The resulting LF is shown in Fig. 8. In spite of this correction, the profile of the LF does not change. The gap at $M_{v} \approx$ 7 and the peak at $M_{v} \approx 12$ persist.
Fig. 5. a) Histogram constructed with the synthetic CMD from Fig. 4, showing the number of stars as a function of the magnitude $R$. The magnitude and color bins are 1.0 and 0.1 , respectively

Fig. 5. b) The same as Fig. 5a, but now with a magnitude bin $=0.5$. The features observed in Fig. 5a remain

\section{White dwarfs candidates}

In the color-magnitude diagrams (see for example Fig. 1a), some blue and very dim stars appear, between $R-I \approx$ 0.2 and 0.4 and $R \approx 23$ and 25 , with photometric errors less than 0.1 magnitude. These candidates are shown in Table 5. All of these objects have a stellar-like profile, as it is indicated by the sharp parameter given by ALLSTAR. If they are cluster members, they could be white dwarfs, which would be in agreement with recent WD observations for NGC 2477 (von Hippel et al. 1995).

If we use Bergeron et al. (1995) calibrations to obtain the effective temperature of these stars, we find that their temperatures are $T_{\text {eff }} \approx 7500 \mathrm{~K}$ and their ages around 1.34 Gyr, which is in accordance with the cluster age. 
Fig. 6. a) CMD showing the stars which correlate with those that appear in the work of Hartwick et al. (1972). With the stars of the main sequence of this figure, we have constructed a relationship among our $R$ and $I$ magnitudes and the $V$ magnitude (see Eq. (4)). We find two more giants not catalogued by Hartwick et al. (1972)

Fig. 6. b) Color-color diagram for the stars which correlate with the work of Hartwick et al. (1972)

Table 5. White dwarfs candidates, as indicated by the position in the color-magnitude diagram. Their $R$ and $I$ magnitudes, and their photometric errors, are shown. Stars can be seen in Plate 3

\begin{tabular}{ccccccc}
\hline$I D$ & $R$ & $\Delta R$ & $I$ & $\Delta I$ & $R-I$ & $\Delta(R-I)$ \\
\hline 623 & 23.183 & 0.019 & 22.882 & 0.024 & 0.301 & 0.031 \\
658 & 23.027 & 0.029 & 22.635 & 0.020 & 0.392 & 0.035 \\
706 & 23.391 & 0.029 & 23.051 & 0.026 & 0.340 & 0.039 \\
766 & 24.007 & 0.024 & 23.780 & 0.048 & 0.227 & 0.054 \\
808 & 24.243 & 0.031 & 23.970 & 0.054 & 0.273 & 0.062 \\
811 & 24.321 & 0.042 & 23.971 & 0.047 & 0.350 & 0.063 \\
830 & 23.714 & 0.024 & 23.457 & 0.065 & 0.257 & 0.069 \\
\hline
\end{tabular}

Fig. 7. LF in visual dereddened magnitude, obtained with Eq. (4) and using extinction coefficients shown in Table 2. We have used a distance modulus for NGC 2477 of $(m-M)_{0}=10.61$ $\pm 0.21(1331 \pm 128 \mathrm{pc})$

Fig. 8. LF with the incompleteness effects due to detection algorithm removed

\section{Low mass star candidates}

Due to the large distance to the cluster, its age, the disc stars contamination and the interstellar absorption, is not easy to find low mass star candidates (LMSC). However, some important steps in that direction can be taken. First of all, the LMSC must be found at the faint red end of the Main Sequence. The second important step is to have an estimate of their masses. The only estimator we may use is the effective temperature for the stars of the lower main sequence, by mean of their colors, which can give only an estimate of their masses. For that purpose we use the linear color-temperature relationship given by Stauffer et al. (1991), as a function of the color $(R-I)_{K}$,

$$
T_{\text {eff }}=-902.3(R-I)_{K}+4151[K] .
$$

The last equation was derived from the Gliese catalog (Gliese \& Jahreiss 1979), This relation is valid for 
Fig. 9. Hertzprung-Russell diagram for some selected stars with a high probability to belong to the cluster. Effective temperatures were calculated ussing the work of Schmidt-Kaler (1982). Open circles are the low mass stars candidates (see Table 6)

spectral types later than $M_{0}\left((R-I)_{K} \geq 0.9\right)$ provided that the colors are previously dereddened (see Tables 2 and 3). The LMSC candidates are shown in Table 6, with their effective temperatures and their $R$ and $I$ magnitudes as well as their corresponding errors. Figure 9 shows the position in the color-magnitude diagram of these LMSC. If the effective temperatures of these stars are close to the temperatures indicated in Table 6 , then their masses must be between 0.1 and $0.5 M_{\odot}$.

\section{Discussion and conclusions}

We have shown that a combination of optical and IR photometry can be used to construct the LF of an open cluster, or more precisely, to discriminate the field stars from cluster ones. This method is useful when the number of field stars in the line of sight of the cluster is no longer negligible. The color diagrams $R-I$ vs. $I-J$ is a good discriminator between cluster and field stars. This result has been established also by Leggett \& Hawkins (1988), who studying the Hyades cluster, concluded that the color $I-J$ is sensitive to changes in the metallicity, making this color a good metallicity indicator.

In spite of NGC 2477 distance $(\approx 1.3 \mathrm{Kpc})$, it has been possible to reach faint absolute magnitudes up to $M_{v} \approx$ 13. The luminosity function we have determined, shows the two well known features found in other works, namely, the Wielen dip (Wielen 1971) at $M_{v} \approx 7$ and probably a maximum at $M_{v} \approx 12$. We found that the LF does not increase for magnitudes greater than $M_{v} \approx 12$. The completeness problem at $M_{v} \approx 12$ is important, preventing us from concluding that it is an unbiased maximum. About the decrease of the number of stars observed at $M_{v} \approx 7$, some authors have suggested that this could be caused by an opacity change in the $\mathrm{H}^{-}$(Kroupa et al. 1992). At the same time, the change of slope between the color-color sequence of the most luminous main sequence stars of the cluster, and the faint stars, could be the product of an increase of $\mathrm{H}^{-}$opacity at $M_{v} \approx 7$, decreasing the luminosity of the stars with magnitudes $M_{v} \geq 7$, therefore the observed number of stars. Our result are not in contradiction with such works. If we adopt the mass luminosity relation of Kroupa et al. (1991), which considers the effects of the $\mathrm{H}_{2}$, the change of slope in our work would appear near $0.6 M_{\odot}$, which is also in agreement with other works (Scalo 1986).

Acknowledgements. This work was a partial fulfilment of the requeriments for the degree of Master at the Universidad de Chile (1993). GG thanks Universidad de Chile for the fellowship No. PG-068-93. MTR thanks FONDECYT grants No. 880-92 and 1950588. We thank the Mac Arthur Foundation, which through the AAAS Starter Grant, allows us to carry on the observations. We would like to thank the referee, Dr. Giovanni Carraro, for his detailed comments on the final version of this paper.

\section{References}

Aspin C., Sandell G., Russell A.P.G., 1994, A\&AS 106, 165

Bahcall J., Flynn C., Gould A., Kirhakos S., 1994, ApJ 435, L51

Bergeron P., Wesemael F., Beauchamp A., 1995, (accepted for publication in PASP)

Carraro G., Ortolani S., 1994, A\&AS 106, 573

Cohen J.G., Frogel J.A., Person S.E., Elias J.H., 1981, ApJ 249, 481

Fahlman G.G., Richer H.B., Searle L., Thompson I.B., 1989, ApJ 343, L49

Fletcher A.B., Stahler S.W., 1994, ApJ 435, 313

Gliese W., Jahreiss H., 1979, A\&AS 38, 423

Hartwick F.D.A., Hesser J.E., McClure R.D., 1972, ApJ 174, 557

von Hippel T., Gilmore G., Jones D.H.P., 1995, MNRAS 273, L39

Janes K.A., Phelps R.L., 1994, AJ 108, 1773

Kroupa P., Tout C.A., Gilmore G., 1991, MNRAS 251, 293

Kroupa P., Tout C.A., Gilmore G., 1992, AJ 103, 1602

Landolt A., 1992, AJ 104, 340

Leggett S.K., Hawkins M.R.S., 1988, MNRAS 234, 1065

Leggett S.K., Harris H.C., Dahn C.C., 1994, AJ 108, 944

Paresce F., de Marchi G., Romaniello M., 1995, ApJ 440, 216

Prosser C.F., 1992, AJ 103, 488

Scalo J.M., 1986, Fund. Cosm. Phys. 11, 1

Schmidt-Kaler T.H., 1982, in Physical Parameter of the stars, Landolt-Bornstein Series, 2b. New York, Springer-Verlag

Stauffer J., Klemola A., Prosser C.F., Probst R., 1991, AJ 101, 980

Stetson P.B., 1987, PASP 9, 191

Thuan T.X., Gunn J.E., 1976, PASP 88, 543

Wielen R., 1971, A\&A 13, 309

Will J.M., Bomans D.J., De Boer K.S., 1995, A\&AS 295, 54 